\title{
Phodopus roborovskii SH101 as a systemic infection model of SARS-CoV-2
}

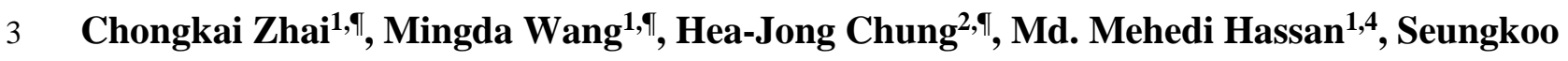

$4 \quad$ Lee $^{3}$, Hyeon-Jin Kim ${ }^{4, *}$ and Seong-Tshool Hong1,*

51 Department of Biomedical Sciences and Institute for Medical Science, Jeonbuk National

6 University Medical School, Jeonju, Jeonbuk 54907, South Korea, 2 Gwangju Center, Korea

7 Basic Science Institute, Gwangju 61751, 3 Department of Anatomic Pathology, School of

8 Medicine, Kangwon National University, Kangwon National University Hospital, 1

9 Gangwondaehak-gil, Chuncheon, Gangwon 24341, South Korea, 4 JINIS BDRD Institute,

10 JINIS Biopharmaceuticals Inc., 224 Wanjusandan-6-ro, Bongdong, Jeonbuk 55315, South

$11 \quad$ Korea.

12 II These authors contributed equally to this work.

13 *hjkim@jinisbio.com (H.-J.K.); seonghong@jbnu.ac.kr (S.-T.H.)

14 Short title: Phodopus roborovskii SH101 as an animal model of SARS-CoV-2 


\section{Abstract}

16 Severe acute respiratory syndrome CoV-2 (SARS-CoV-2) is currently causing a worldwide threat

17 with its unusually high transmission rates and rapid evolution into diverse strains. Unlike typical

18 respiratory viruses, SARS-CoV-2 frequently causes systemic infection by breaking the boundaries

19 of the respiratory systems. The development of animal models recapitulating the clinical manifestations of COVID-19 is of utmost importance not only for the development of vaccines and antivirals but also for understanding the pathogenesis. However, there has not been developed an animal model for systemic infection of SARS-CoV-2 representing most aspects of the clinical manifestations of COVID-19 with systemic symptoms. Here we report that a hamster strain of Phodopus roborovskii SH101, a laboratory inbred hamster strain of $P$. roborovskii, displayed most symptoms of systemic infection upon SARS-CoV-2 infection as in the case of the human counterpart, unlike current COVID-19 animal models. P. roborovskii SH101 post-infection of SARS-CoV-2 represented most clinical symptoms of COVID-19 such as snuffling, dyspnea, cough, labored breathing, hunched posture, progressive weight loss, and ruffled fur, in addition to high fever following shaking chills. Histological examinations also revealed a serious rightpredominated pneumonia as well as slight organ damages in the brain and liver, manifesting systemic COVID-19 cases. Considering the merit of a small animal as well as its clinical manifestations of SARS-CoV-2 infection in human, this hamster model seems to provide an ideal tool to investigate COVID-19.

\section{Author summary}

35 Although the current animal models supported SARS-CoV-2 replication and displayed varying 
to the respiratory systems of these animals, including hACE2 transgenic mice, hamsters, ferrets, fruit bats, guinea pigs, African green monkey, Rhesus macaques, and Cynomolgus macaques. While these animal models can be a modest model for the respiratory infection, there is a clear limit for use them in the study of COVID-19 that also displays multiple systemic symptoms. Therefore, the development of an animal model recapitulating COVID-19-specific symptoms such as the right-predominated pneumonia would be the utmost need to overcome the imminent threat posed by COVID-19. We identified a very interesting hamster strain, Phodopus roborovskii SH101, which mimics almost all aspects of the clinical manifestations of COVID-19 upon SARSCoV-2 infection. Unlike the current animal models, SARS-CoV-2-infected P. roborovskii SH101 not only displayed the symptoms of respiratory infection but also clinical manifestations specific to human COVID-19 such as high fever following shaking chills, serious right-predominated pneumonia, and minor organ damages in the brain and liver.

\section{Introduction}

The emergence of COVID-19 upon the infection of SARS-CoV-2 in 2019 poses a mounting threat to the world. In COVID-19, the infection of SARS-CoV-2 in the respiratory system causes fever, shaking chills, headache, and fatigue followed by respiratory symptoms such as cough, sneeze, sore throat, chest pain, atypical pneumonia, etc [1,2]. The clinical features of COVID-19, however, are not limited to the consequences of a respiratory infection [3]. Unlike a typical respiratory infection, SARS-CoV-2 infects the organs other than the respiratory system from the beginning or subsequent to respiratory infection, leading to diarrhea, loss of sense of smell or taste, neuroinflammation manifested as encephalitis, meningitis, acute cerebrovascular disease, and Guillain Barré Syndrome (GBS), multisystem inflammatory syndrome, etc [4-6]. More interestingly, atypical pneumonia by SARS-CoV-2 is observed with heavy predominance in the 
right lung [7]. The right-over-left predominance in pneumonia is one of the hallmarks of COVID19 disease [8,9]. As the consequences of these systemic infection, patients recovered from severe or even mild COVID-19 are frequently suffering from fatigue, heart palpitations, changes in lung function, muscle weakness, memory loss, concentration, brain fog, depression, anxiety, etc [10-12].

The mutation rate in RNA virus such as SARS-CoV-2 is dramatically high, up to a thousand times higher than that of DNA virus, contributing to rapid evolution into its variants. Considering the nature of RNA genome and the number of already happened cases of outbreak, the emergence of new strains from furtively circulating SARS-CoV-2 even after herd immunity by vaccination seems to be inevitable. In fact, the world is already observing the emergence of multiple variants of SARS-CoV-2. Given the number of already spread outbreak cases, an appropriate surrogate for human COVID-19 is needed to overcome the imminent threats of COVID-19 from a future emergence of variant strains. Moreover, animal models could help shed light on important aspects of human COVID-19 in ways that are not easily addressed or feasible in humans, such as how SARS-CoV-2 causes systemic infection.

After the outbreak of COVID-19, various animal models for COVID-19 have been developed. The current animal models, such as hACE2 transgenic mice, hamsters, ferrets, fruit bats, guinea pigs, African green monkey, Rhesus macaques, and Cynomolgus macaques, supported SARSCoV-2 replication and displayed varying degrees of illness when the virus was delivered into the respiratory tract of these animals [13-17]. However, the infections of SARS-CoV-2 in these animals were mainly limited to the respiratory systems. Since these animal models mostly recapitulated the infections of the respiratory system, each of these animals has a limited utility in the study of COVID-19 [18-22]. Therefore, the development of an animal model recapitulating 
systemic infection, including COVID-19-specific facet such as the right-predominated pneumonia, would be the utmost need to overcome the imminent threat posed by COVID-19.

In this work, we identified a laboratory-inbred strain of Phodopus roborovskii, $P$. roborovskii SH101, representing the systemic infection of human COVID-19. In addition to systemic infection, the infection of $P$. roborovskii SH101 with SARS-CoV-2 caused almost all clinical symptoms represented by human COVID-19, including high fever, shaking chills, serious right-predominated pneumonia, and the viral distribution pattern as well as evident respiratory and behavioral symptoms.

\section{Results}

\section{A hamster strain recapitulating COVID-19 after SARS-CoV-2 infection was identified}

To develop an ideal animal model of COVID-19, we screened several hundred strains of mice, rats, guinea pigs, and hamsters, and identified a hamster strain, Phodopus roborovskii SH101, which was highly sensitive to SARS-CoV-2 infection. P. roborovskii SH101 (abbreviated as SH101) is a laboratory-inbred hamster strain with prominent white patches above eyes and at the base of ears (Fig 1A, B). The average adult body weights of SH101 were about $21 \mathrm{~g}$ for males and $20 \mathrm{~g}$ for females, respectively.

As in the case of COVID-19, respiratory symptoms were immediately noticed in the SH101 hamsters infected with SARS-CoV-2 (Video S1 S4). The hamsters showed clear signs of respiratory symptoms such as snuffling, dyspnea, cough, labored breathing, ruffled fur, and sneeze. Along with the respiratory symptoms, the very active behavior otherwise typical for the hamsters was dramatically reduced while hunched posture was observed starting from 1-day post-infection (dpi). Other than these clinical manifestations associated with the severe respiratory and systemic infection, some hamsters showed shaking chills (Video S3) after 2 dpi which resembled the 
106

107

108

109

110

111

112

113

114

115

116

117

118

119

120

121

122

123

124

shaking chills of human patients of COVID-19. Also, progressive and significant weight loss had been observed from 2 to 4 dpi (Fig 1C, D). All individuals were terminally ill, and the mortality rate of the SH101 hamsters was $83 \%$ by 4 dpi.

Most interestingly, a unique uneven distribution of pneumonia was noticed immediately by gross examination of the lung specimens (Fig 1E). The dissected lungs of the infected SH101 hamsters showed the right-predominated pneumonia just like as in the case of COVID-19. It was remarkably interesting to note the right-predominated pneumonia of the hamster because one of the most peculiar clinical manifestations of COVID-19 is the right-over-left predominated pneumonia [23-25].

\section{P. roborovskii SH101 infected with SARS-CoV-2 progressed similarly with human COVID-} 19

Although the induction of fever in COVID-19 is an essential hallmark of SARS-CoV-2 infection, none of the current animal models, including primates, showed an induced fever by SARS-CoV2 infection. In this study, the body temperature measurement by an infrared thermographic method revealed the induction of fever in SH101 hamsters immediately after infection of SARS-CoV-2 (Fig 2). The elevated body temperatures of the infected SH101 hamsters were sharply dropped as the infection progressed to the terminal stage. Being one of the cardinal features in COVID-19 $[26,27]$, the fever induction after SARS-CoV-2 infection seems to indicate that the SH101 hamsters simulate COVID-19 symptoms most closely among animal models of COVID-19.

Another unusual feature of human COVID-19 is the widespread thrombosis of small and large vessels, contributing to morbidity and mortality ${ }^{28-30}$. It was also known that fibrinolysis was elevated in human COVID-19 due to systemic multiorgan thrombosis by SARS-CoV-2 infection [31,32]. The levels of fibrin degradation products, D-dimer and FDP, were elevated in the plasmas 
of the SH101 hamsters infected with SARS-CoV-2 (Fig 3A D), suggesting the occurrence of systemic infection in the hamsters as in the case of human COVID-19.

We next quantitated SARS-CoV-2 in the SH101 hamsters by quantitative RT-PCR after reverse transcription (RT-qPCR). The primary organs were collected from 3 randomly assigned individuals in each group at 2 and 4 dpi for analysis. High levels of viral RNA were detected in the homogenates of the lung and trachea, whereas lower levels were detected in the brain, stomach, intestine, liver, blood, heart, kidney, and spleen (Fig 3E, F). The detection of the viral RNAs in all organs from the SH101 hamsters confirmed serious systemic infection caused by SARS-CoV-2.

\section{The histological examinations of $P$. roborovskii SH101 infected with SARS-CoV-2 revealed} severe inflammation in the lungs and minor pathologies in the liver and brain

After observing that $P$. roborovskii SH101 infected with SARS-CoV-2 closely represented the clinical manifestations of COVID-19 in humans, we examined the histopathological changes in each organ of the hamsters (Fig 4). Slides were carefully evaluated by a pathologist blinded to the treatment groups. As shown in Fig 4A, pathological examinations revealed severe inflammatory lesions in the lungs of the infected SH101 hamsters starting from 2 dpi. The lesions in the lungs were the typical features of severe viral pneumonia, as shown in diffuse alveolar damage (DAD) with hyaline membrane formation and type II pneumocyte desquamation at 2 dpi. The DAD pattern showed a mixture of exudative and proliferative features. The inflammatory lung lesions were extended across larger areas without any alveolar spaces due to the severity of inflammation at the last day, 4 dpi.

In addition to the pulmonary injury, slight pathological damages were also observed in the livers and brains of the infected SH101 hamsters (Fig 4B). The pathological damages were observed in 4 out of the 6 livers at 2 dpi and 6 out of the 6 livers at 4 dpi, along with histological 
examinations. Multifocal fatty changes and portal lymphocytic infiltration observed in the livers are shown in Fig 4B. The hepatic injury also strongly supported that the SH101 hamsters infected with SARS-CoV-2 closely mimicked COVID-19 in human [33,34].

In brain specimens, like the liver, we also observed a focal infiltration of lymphocyte and/or subarachnoid hemorrhage in 4 out of the 6 brains at 2 dpi and 4 out of the 6 brains at 4 dpi (Fig 4C), suggesting possible neuroinflammation just like as in the case of COVID-19 disease [35,36]. Moreover, extravasation of red blood cells and hemosiderin pigments with focal infiltration of lymphocytes were also observed in the damaged brains. In terms of frequent neurological complications in COVID-19, the neurological damages (Fig 4C) in the infected SH101 hamsters also recapitulated the important clinical features of COVID-19. Despite high levels of SARS-CoV2, the trachea was not damaged by SARS-CoV-2 infection, demonstrating another piece of similarity to COVID-19 [37]. The undamaged trachea despite the high viral titers implies that the trachea may function as an exhauster in the hamsters like the human counterpart. Other than these organs, no obvious histopathological changes were observed in the stomach, intestine, heart, kidney, and spleen (Fig S1).

\section{Comparison of $P$. roborovskii SH101 with the hACE2 transgenic mouse and Syrian hamster}

\section{to study COVID-19}

Among the various animal species investigated as an animal model for COVID-19, the hACE2 transgenic mice and Syrian hamsters are the most widely-used animal models to study human COVID-19. However, despite their popularity in COVID-19 studies, infections of the hACE2 transgenic mice or Syrian hamsters with SARS-CoV-2 resulted in infection mostly in the respiratory system. Considering their broad use in COVID-19 researches, these two animal models were analyzed in comparison with the SH101 hamster after SARS-CoV-2 infection. In agreement 
175

176

177

178

with previous studies, our experiments using hACE2 transgenic mice and Syrian hamsters confirmed their sensitivity to SARS-CoV-2 (Fig 5, 6 and Fig S2, S3) but failed to observe representative symptoms of COVID-19 such as fever induction, shaking chills, the right-over-left predominated pneumonia, etc.

The fever induction and shaking chills are the most common and primary symptoms of COVID-19 that can be observed even in mild cases [26,27]. For the first time, we were able to show the cardinal symptoms of COVID-19, fever induction, shaking chills, and respiratory problems, in a SARS-CoV-2-infected animal just like in the human counterpart (Fig 1 4, Fig S4 S7 and Video S1 S4), but not in other models (Fig 5, 6).

Both hACE2 transgenic mice and Syrian hamster with SARS-CoV-2 infection demonstrated certain degree of organ damages, heavily damaged lungs and slightly damaged livers but no detectable pathology in the brains (Fig 6A D and Fig S3). Interestingly, the viral RNA copies in the lungs of SH101, the hACE2 transgenic mice, and Syrian hamsters were not much different, 6.4 \pm 0.4 (4 dpi), $5.7 \pm 0.3$ (4 dpi), and $5.6 \pm 0.2$ (4 dpi), respectively (Fig 6E, F). The dramatic differences of clinical manifestations between current models and SH101 despite of the similar range of virus titers (Fig 3E, F and Fig 6E, F) suggests that a physiological makeup of $P$. roborovskii SH101 would be different from those of the other two animals.

It has been well studied that young animals were significantly less sensitive to SARS-CoV-2 $[38,39]$. While hACE2 transgenic mice and Syrian hamsters displayed age-dependent sensitivity, SH101 hamsters as young as two-month-old were able to show serious symptoms by SARS-CoV2 infection (Fig S4 S7). The symptomatic severities of the young hamsters assessed by the organ damages were comparable to those of adults (Fig 4 and Fig S1, S5, S6). The virus titers of the young SH101 were $7.0 \pm 0.3$ in the lung at 4 dpi (Fig S7) like those of adults (Fig 3E, F). Despite 
the general similarity between the young and adult hamsters, SARS-CoV-2 infection led to a $100 \%$ mortality rate in the young SH101 hamsters by 4 dpi.

\section{Discussion}

Multiple animal species have been investigated as an animal model for COVID-19 since the onset of the pandemic $[38,39]$. Among the developed animal models to study COVID-19, the hACE2 transgenic mice and Syrian hamsters are the most favorable animal models currently because of the merit of easy handling of small animals $[13,15]$. Despite of their popularity in the COVID-19 studies, the common use of these two animals for SARS-CoV-2 infection needs to be reconsidered due to their limits in the implementation of human COVID-19. This work confirmed that SARSCoV-2 infections are mostly localized in the respiratory systems of the hACE2 transgenic mice and Syrian hamsters (Fig 5, 6 and Fig S2, S3) in agreement with previous studies [13,15]. COVID19 , however, is known to display a various degree of systemic infection in addition to localized respiratory infection, even in mild cases, indicating that the hACE2 transgenic mice and Syrian hamsters are not ideal models to study human COVID-19.

The repertory of clinical presentations of COVID-19 is far exceeding respiratory symptoms, including diarrhea, loss of sense of smell or taste, neuroinflammation manifested as encephalitis, meningitis, acute cerebrovascular disease, GBS, multisystem inflammatory syndrome, thrombosis, hyperfibrinolysis, etc [4,5]. Given the nature of the systemic infection of COVID-19, an ideal COVID-19 animal model should represent not only most respiratory infection but also other systemic infection observed in human COVID-19. Unfortunately, none of the current COVID-19 animal models reproduce the salient clinical and pathological features of human COVID-19 22. Even primate models, the closest animal models to humans, did not reproduce the clinical symptoms from the systemic infection of human COVID-19 [16,38]. It is therefore pivotal to 
221 develop an animal model for the systemic infection replicating most clinical aspects of human

222 COVID-19. In this regard, $P$. roborovskii SH101 would be an ideal animal model for evaluating

223 antiviral therapeutic agents and vaccines, as well as understanding the pathogenesis of human

224 COVID-19. Interestingly, $P$. roborovskii SH101 infected with SARS-CoV-2 recapitulated the very interesting COVID-19-specific facet, the right-predominated pneumonia (Fig 1E), which are uniquely observed in human COVID-19.

P. roborovskii SH101 not only reproduced the salient clinical and pathological features of human COVID-19 but also demonstrated unusual rapid progression once infected. The SARSCoV-2-infected hamsters are observed with fever induction early on $1 \sim 2$ dpi, followed by rapid progression into the terminal stage to death by $3 \sim 4$ dpi (Fig 2). The unusual rapid progression in addition to clinical presentations would provide an ideal animal model to investigate disease mechanisms and to develop drugs and vaccines for COVID-19. These characteristics of the SH101 hamster model seem to eliminate the necessity for the primate models of COVID-19.

SARS-CoV-2 is an RNA virus that quickly evolves into various strains by rapid mutations. In fact, multiple strains of SARS-CoV-2 have already emerged [40], and thus the development of animal models recapitulating most of the clinical manifestations of COVID-19 would be of utmost importance not only for overcoming COVID-19 but also for understanding the pathogenesis. Further genome analyses of $P$. roborovskii SH101 in comparison with SARS-CoV-2-resistant hamsters or with SARS-CoV-2-susceptible hamsters for the local respiratory infection, such as Syrian hamsters, would provide critical clues for the understanding of human COVID-19.

\section{Materials and methods}

\section{Ethics approval}


All procedures involving the mice and hamsters were accredited with the approval of the Institutional Animal Care and Use Committee (IACUC), in compliance with the guidelines of the Ethics Committee of Jeonbuk National University Laboratory Animal Center Guidelines on the Care and Use of Animals for Scientific Purposes. All animal experiments in this study were in accordance with the ARRIVE guidelines and checklist. All the animals used in this research were handled in a manner consistent with CDC/ABSA/WHO guidelines for the prevention of human infection with the SARS-CoV-2 virus.

\section{Viruses and cells}

The SARS-CoV-2 strain HB-01 was obtained from the National Culture Collection for Pathogens (NCCP) of the Korea Disease Control and Prevention Agency (KDCA). The complete genome for this SARS-CoV-2 has been submitted to GISAID (identifier: BetaCoV/Wuhan/IVDC-HB01/2020|EPI_ISL_402119), and deposited in the China National Microbiological Data Center (accession number NMDC10013001 and genome accession number MDC60013002-01). Preparation of seed SARS-CoV-2 stocks and isolation of the virus were performed in Vero cells, which were maintained in Dulbecco's modified Eagle's medium (DMEM) supplemented with 10\% fetal bovine serum (FBS), $100 \mathrm{IU} / \mathrm{ml}$ penicillin, and $100 \mu \mathrm{g} / \mathrm{ml}$ streptomycin, and incubated at $37^{\circ} \mathrm{C}, 5 \% \mathrm{CO}_{2}$

\section{Animal experiments}

Six-week-old hACE2 transgenic mice (K18-hACE2 strain) having the human angiotensin I converting enzyme (peptidyl-dipeptidase A) 2 (hACE2) gene under the human cytokeratin 18 (K18) promoter [41] on chromosome $2(99,209,508-99,220,724)$ and Syrian hamsters (Mesocricetus auratus) were purchase from Alpha biochemicals Co 
265 (http://alphabiochemicals.com). P. roborovskii SH101 was a laboratory inbred hamster strain

266 maintained in the Jinis Biopharmaceuticals Inc. (Wanju, Jeonbuk, Republic of Korea). We

267 deposited P. roborovskii SH101 for distribution in Alpha biochemicals Co.

For animal experiments, three animals of each animal species were housed in one cage and

2 solution containing $10^{5} \mathrm{TCID}_{50}$ or mock (DMEM) was inoculated intranasal by using a pipette.

The food consumption of each mouse group was monitored daily. For blood profiling and behavioral tests, animals were randomly assigned to each treatment group. Particularly, we declare that blinding was employed during animal allocation and data collection.

\section{Body temperature measurement}

The body temperatures of the animals were measured by a high-precision thermal photographic method $[42,43]$. Each of the whole animal bodies was photographed by FLIR thermal imaging

281 body temperature. The highest temperature spot on the chest, close to the site of the lung, was

282 allocated in each mouse to determine the body temperature. All data are presented as the mean \pm 283 standard deviation and were compared using paired Student's t-tests.

\section{D-dimer and Fibrin degradation products (FDPs) Analysis}


The hypercoagulable state of the SARS-CoV-2-infected hamsters was analyzed daily by D-dimer and FDPs assays during the experimental period. Whole blood was collected in $1.5 \mathrm{ml}$ Eppendorf micro-centrifuged tube by cardiac puncture. Blood serum was separated by centrifugation at 2,500 $\times \mathrm{g}$ for $10 \mathrm{~min}$ and stored at $25^{\circ} \mathrm{C}$ until used. The D-dimer and FDPs concentrations of serum samples were determined by the double antibody sandwich method using mouse D-dimer and mouse FDPs ELISA kits (Sunlong Biotech, Hangzhou, Zhejiang, China) as described previously [44]. Briefly, $20 \sim 23$ times diluted sera with the dilution buffer were used to quantitate D-dimer or FDPs in a micro-ELISA strip plate pre-coated with mouse anti-D-dimer or anti-FDPs monoclonal antibody. The concentrations of D-dimer and FDPs were calculated from the OD values of each sample using a standard curve.

\section{Preparation of total RNAs from the primary organs}

After euthanized each mouse of the experimental groups, each mouse was dissected to isolate primary organs; lung, brain, liver, pancreas, kidney, stomach, trachea, spleen, small intestine, heart, and colon. Tissue homogenates $(100 \mathrm{mg} / \mathrm{ml})$ were prepared by homogenizing perfused organs using an electric homogenizer for $2 \mathrm{~min} 30 \mathrm{~s}$ in DMEM. The homogenates were centrifuged at $3,000 \times \mathrm{rpm}$ for $10 \mathrm{~min}$ at $4^{\circ} \mathrm{C}$. The supernatants were collected and stored at $-80^{\circ} \mathrm{C}$ until virus quantifications.

\section{Quantitation of SARS-CoV-2 titers}

Total RNA was extracted from the supernatants of the organ homogenates using the RNeasy Mini Kit (QIAGEN, Hilden, Germany), and reverse transcription was performed using the PrimerScript RT Reagent Kit (TaKaRa, Japan) following the manufacturers' instructions. RT-qPCR reactions were performed using the PowerUp SYBG Green Master Mix Kit (Applied Biosystems, Waltham, 
MA, USA), in which samples were processed in duplicate using the following cycling protocol: $50^{\circ} \mathrm{C}$ for $2 \mathrm{~min}, 95^{\circ} \mathrm{C}$ for $2 \mathrm{~min}$, followed by 40 cycles at $95^{\circ} \mathrm{C}$ for $15 \mathrm{~s}$ and $60^{\circ} \mathrm{C}$ for $30 \mathrm{~s}$, and then $95^{\circ} \mathrm{C}$ for $15 \mathrm{~s}, 60^{\circ} \mathrm{C}$ for $1 \mathrm{~min}, 95^{\circ} \mathrm{C}$ for $45 \mathrm{~s}$. The primer sequences used for RT-qPCR is targeted against the envelope (E) gene of SARS-CoV-2 and are as follows: forward: 5'products were verified by sequencing using the dideoxy method on an ABI 3730 DNA sequencer database. The SYBR green real-time PCR standard curve was generated by serial ten-fold dilutions of recombinant plasmid with a known copy number (from $7 \times 10^{7}$ to $7 \times 10^{1}$ copies per $\mu 1$ ). These

317 dilutions were tested and used as quantification standards to construct the standard curve by 318 plotting the plasmid copy number against the corresponding threshold cycle values $(\mathrm{Ct})$. Results 319 were expressed as $\log _{10}$-transformed numbers of genome equivalent copies per $\mathrm{ml}$ of sample. The $\mathrm{Ct}$ values of each sample were used to quantitate the virus titers by using the standard curve.

\section{Histology}

About halves of the primary organs (lung, brain, liver, pancreas, kidney, stomach, trachea, spleen, small intestine, heart, and colon) were isolated from 3 adult postmortem P. roborovskii SH101 at 2 dpi (euthanized hamsters) and 4 dpi (hamster carcasses at terminal stage), respectively. The same primary organs were isolated for histological examination from 2-month-old P. roborovskii SH101 carcasses at $4 \mathrm{dpi}$, euthanized hACE2 transgenic mice at $7 \mathrm{dpi}$, and euthanized Syrian golden 
were air-dried and baked overnight at $65^{\circ} \mathrm{C}$. The organ sections were stained with Hematoxyline and Eosin $(\mathrm{H} \& \mathrm{E})$ with standard staining procedure $[45,46]$. The stained tissue images were observed in Apero ScanScope FL (Leica Biosystems, Germany).

\section{Statistical analysis}

The statistical significance of SARS-CoV-2-infected and mock-treated samples was assessed by a one-way ANOVA multiple comparisons test. Statistical analyses were performed using GraphPad Prism 5 (GraphPad Software, La Jolla, CA, USA). A p-value less than 0.05 was considered statistically significant.

\section{Supporting Information}

\section{S1 files. Figure S1 S7containing (PDF)}

Fig S1: The histological images of the primary organs of P. roborovskii SH101 post-infection of SARS-CoV-2. Fig S2: The body weight changes for the hACE-transgenic mice and Syrian golden hamsters post-infection of SARS-CoV-2. Fig S3: The histological examination results of the primary organs showing no pathological damages of the hACE-transgenic mice and Syrian golden hamsters post-infection of SARS-CoV-2. Fig S4: The changes of the body weight and the body temperatures of young $P$. roborovskii SH101 post-infection of SARS-CoV-2. Fig S5: The results of histological examination of the primary organs showing pathologies of young $P$. roborovskii SH101 post-infection of SARS-CoV-2. Fig S6: The histological examination results of the primary organs showing no pathological damages of young $P$. roborovskii SH101 post-infection of SARSCoV-2. Fig S7: SARS-CoV-2 replication in young $P$. roborovskii SH101 after SARS-CoV-2 infection. 
S2 files. Video S1 S4 containing (ZIP)

Video S1: General behavioral symptoms of male P. roborovskii SH101 infected with SARS-CoV-

2. Video S2: The sneezing symptom of P. roborovskii SH101 infected with SARS-CoV-2. Video

S3: The shaking chills of $P$. roborovskii SH101 infected with SARS-CoV-2. Video S4: General

\section{Author Contributions}

357 Conceptualization: Seong-Tshool Hong

358 Data curation: Chongkai Zhai, Mingda Wang

359 Formal analysis: Chongkai Zhai, Mingda Wang, Hea-Jong Chung, Md. Mehedi Hassan

360 Funding acquisition: Seong-Tshool Hong

361 Investigation: Seong-Tshool Hong

362 Methodology: Chongkai Zhai, Mingda Wang, Hea-Jong Chung, Md. Mehedi Hassan

363 Project administration: Chongkai Zhai, Mingda Wang

364 Resource: Chongkai Zhai, Mingda Wang, Hea-Jong Chung, Md. Mehedi Hassan

365 Software: Chongkai Zhai, Mingda Wang, Hea-Jong Chung, Md. Mehedi Hassan

366 Supervision: Seong-Tshool Hong

367 Validation: Chongkai Zhai, Mingda Wang

368 Visualization: Chongkai Zhai, Mingda Wang, Seungkoo Lee

369 Writing-original draft: Seong-Tshool Hong, Hyeon-Jin Kim

370 Writing-review \& editing: Seong-Tshool Hong, Hea-Jong Chung

\section{$371 \quad$ Financial Disclosure Statement}


This research was supported by JINIS BDRD Research Institute of JINIS Biopharmaceuticals Inc. (Initial of authors who received fund: S. H.). The funder had no role in study design, data collection and analysis, decision to publish, or preparation of the manuscript.

\section{References}

1. Ahmad S, Shoaib A, Ali S, Alam S, Alam N, Ali M, et al. Epidemiology, risk, myths, pharmacotherapeutic management and socio economic burden due to novel COVID-19: A recent update. Research Journal of Pharmacy and Technology. 2020; 13: 4435-4442.

2. Yasuhara J, Kuno T, Takagi H, Sumitomo N. Clinical characteristics of COVID-19 in children: A systematic review. Pediatric pulmonology. 2020; 55: 2565-2575.

3. Fajnzylber J, Regan J, Coxen K, Corry H, Wong C, Rosenthal A, et al. SARS-CoV-2 viral load is associated with increased disease severity and mortality. Nature communications. 2020; 11: 1-9.

4. Ellul MA, Benjamin L, Singh B, Lant S, Michael BD,Easton A, et al. Neurological Associations of COVID-19. Lancet Neurol. 2020; 19: 767-783.

5. Song Y, Liu P, Shi XL, Chu YL, Zhang J, Xia J, et al. SARS-CoV-2 induced diarrhea as onset symptom in patient with COVID-19. Gut. 2020; 69: 1143-1144.

6. Spinato G, Fabbris C, Polesel J, Cazzador D, Borsetto D, Hopkins C, et al. Alterations in smell or taste in mildly symptomatic outpatients with SARS-CoV-2 infection. JAMA. 2020; 323: 2089-2090.

7. Yang W, Sirajuddin A, Zhang X, Liu G, Teng Z, Zhao S, et al. The role of imaging in 2019 novel coronavirus pneumonia (COVID-19). European Radiology. 2020; 30: 4874-4882. 
8. Bernheim A, Mei X, Huang M, Yang Y, Fayad ZA, Zhang N, et al. Chest CT findings in coronavirus disease-19 (COVID-19): relationship to duration of infection. Radiology. 2020; 295: 685-691.

9. Yoon SH, Lee KH, Kim JY, Lee YK, Ko H, Kim KH, et al. Chest radiographic and CT findings of the 2019 novel coronavirus disease (COVID-19): analysis of nine patients treated in Korea. Korean J Radiol. 2020; 21: 494-500.

10. Ahmad T, Khan M, Haroon, Musa TH, Nasir S, Hui J, et al. COVID-19: Zoonotic aspects. Travel Med Infect Dis. 2020; 36: 101607.

11. Michelen M, Manoharan L, Elkheir N, Cheng V, Dagens D, Hastie C, et al. Characterising long-term covid-19: a rapid living systematic review. medRxiv. 2020; doi: https://doi.org/10.1101/2020.12.08.20246025.

12. Zheng YY, Ma YT, Zhang JY, Xie X. COVID-19 and the cardiovascular system. Nature Reviews Cardiology. 2020; 17: 259-260.

13. Bao L, Deng W, Huang B, Gao H, Liu J, Ren L, et al. The pathogenicity of SARS-CoV-2 in hACE2 transgenic mice. Nature. 2020; 583: 830-833.

14. Chandrashekar A, Liu J, Martinot AJ, McMahan K, Mercado NB, Peter L, et al. SARSCoV-2 infection protects against rechallenge in rhesus macaques. Science. 2020; 369: $812-817$.

15. Imai M, Iwatsuki-Horimoto K, Hatta M, Loeber S, Halfmann PJ, Nakajima N, et al. Syrian hamsters as a small animal model for SARS-CoV-2 infection and countermeasure development. PNAS. 2020; 117: 16587-16595.

16. Melin AD, Janiak MC, Marrone F, Arora PS, Higham JP. Comparative ACE2 variation and primate COVID-19 risk. BioRxiv. 2020; doi: 10.1101/2020.04.09.034967. 
17. Shi J, Wen Z, Zhong G, Yang H, Wang C, Huang B, et al. Susceptibility of ferrets, cats, dogs, and other domesticated animals to SARS-coronavirus 2. Science. 2020; 368: $1016-1020$.

18. Chen W. Promise and challenges in the development of COVID-19 vaccines. Human Vaccines \& Immunotherapeutic. 2020; 16: 2604-2608.

19. Lakdawala SS, Menachery VD. The search for a COVID-19 animal model. Science. 2020; 368: 942-943.

20. Pandey K, Acharya A, Mohan M, Ng CL, Reid SP, Byrareddy SN. Animal Models for SARS-CoV-2 research: A Comprehensive Literature Review. Transbound Emerg Dis 2020; doi: 10.1111/tbed.13907.

21. Sia SF, Yan LM, Chin AWH, Fung K, Choy KT, et al. Pathogenesis and transmission of SARS-CoV-2 in golden hamsters. Nature 2020; 583: 834-838.

22. Ulrich L, Wernike K, Hoffmann D, Mettenleiter TC, Beer M. Experimental infection of cattle with SARS-CoV-2. Emerging infectious diseases. 2020; 26: 2979.

23. Nagpal P, Narayanasamy S, Vidholia A, Guo J, Shin KM, Lee CH, et al. Imaging of COVID-19 pneumonia: Patterns, pathogenesis, and advances. Br J Radiol. 2020; 93: 20200538.

24. Rousan LA, Elobeid E, Karrar M, Khader Y. Chest X-ray findings and temporal lung changes in patients with COVID-19 pneumonia. BMC Pulmonary Medicine. 2020; 20: 1-9.

25. Zantah M, Castillo ED, Townsend R, Dikengil F, Criner GJ. Pneumothorax in COVID-19 disease-incidence and clinical characteristics. Respiratory Research. 2020; 21: 1-9. 
26. Hassan SA, Sheikh FN, Jamal S, Ezeh JK, Akhtar A. Coronavirus (COVID-19): a review of clinical features, diagnosis, and treatment. Cureus. 2020; 12.

27. Tang D, Comish P, Kang R. The hallmarks of COVID-19 disease. Plos Pathogens. 2020; 16: e1008536.

28. Gu SX, Tyagi T, Jain K, Gu VW, Lee SH, Hwa JM, et al. Thrombocytopathy and endotheliopathy: crucial contributors to COVID-19 thromboinflammation. Nat Rev Cardiol. 2020;19: 1-16.

29. Merrill JT, Erkan D, Winakur J, James JA. Emerging evidence of a COVID-19 thrombotic syndrome has treatment implications. Nat Rev Rheumatol. 2020; 16: 581-589.

30. Rapkiewicz AV, Mai X, Carsons SE, Pittalug S, Kleiner DE, Berger JS, et al. Megakaryocytes and platelet-fibrin thrombi characterize multi-organ thrombosis at autopsy in COVID-19: a case series. EClinicalMedicine. 2020; 24: 100434.

31. Eljilany I, Elzouki AN, D-Dimer, Fibrinogen, and IL-6 in COVID-19 Patients with Suspected Venous Thromboembolism: A Narrative Review. Vascular health and risk management. 2020; 16: 455.

32. Srivastava S, Garg I, Bansal A, Kumar B. COVID-19 infection and Thrombosis. Clinica Chimica Acta. 2020; 510: 344-346.

33. Zhang C, Shi L, Wang FS. Liver injury in COVID-19: management and challenges. The lancet Gastroenterology \& hepatology. 2020; 5: 428-430.

34. Xu L, Liu J, Lu M, Yang D, Zheng X. Liver injury during highly pathogenic human coronavirus infections. Liver International. 2020; 40: 998-1004.

35. Iadecola C, Anrather J, Kamel H. Effects of COVID-19 on the nervous system. Cell. 2020; 183: $16-27$. 
36. Matschke J, Lütgehetmann M, Hagel C, Sperhake JP, Schröder AS, Edler C, et al. Neuropathology of patients with COVID-19 in Germany: a post-mortem case series. The Lancet Neurology. 2020; 19: 919-929.

37. Sun SH, Chen Q, Gu HJ, Yang G, Wang YX, Huang XY, et al. A mouse model of SARSCoV-2 infection and pathogenesis. Cell Host Microbe. 2020; 28: 124-133.

38. Heegaard PM, Sturek M, Alloosh M, Belsham GJ. Animal Models for COVID-19: More to the Picture Than ACE2, Rodents, Ferrets, and Non-human Primates. A Case for Porcine Respiratory Coronavirus and the Obese Ossabaw Pig. Frontiers in Microbiology. 2020; 11: 2333.

39. Muñoz-Fontela C, Dowling WE, Funnell SGP, Gsell PS, Riveros-Balta AX, Albrecht R A, et al. Animal models for COVID-19. Nature 2020, 586, 509-515.

40. Dorp L, Acman M, Richard D, Shaw LP, Ford CE, Ormond L, et al. Emergence of genomic diversity and recurrent mutations in SARS-CoV-2. Infect Genet Evol. 2020; 83: 104351.

41. McCray Jr PB, Pewe L, Wohlford-Lenane C, Hickey M, Manzel L, Shi L, et al. Lethal infection of K18-hACE2 mice infected with severe acute respiratory syndrome coronavirus. J. Virol. 2007; 81: 813-821.

42. Chung HJ, Kim HJ, Hong ST. Iron-dextran as a thermosensitizer in radiofrequency hyperthermia for cancer treatment. Applied Biological Chemistry. 2019; 62: 1-9.

43. Chung HJ, Lee HK, Kwon KB, Kim HJ, Hong ST. Transferrin as a thermosensitizer in radiofrequency hyperthermia for cancer treatment. Scientific reports. 2018; 8: 1-11.

44. Hassan MM, Sharmin S, Kim HJ, Hong ST. Identification and Characterization of Plasmin-Independent Thrombolytic Enzymes. Circulation Research. 2021; 128: 386-400. 

boosts motor coordination and physical strength. Scientific reports. 2016; 6: 23958. virus polymerase in cirrhosis, hepatocellular carcinoma, and steatosis. FEBS open bio. 
A

$$
\text { P. roborovskii SH101 (Female) }
$$

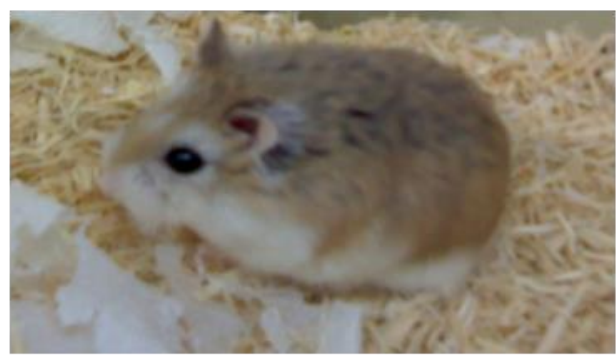

C

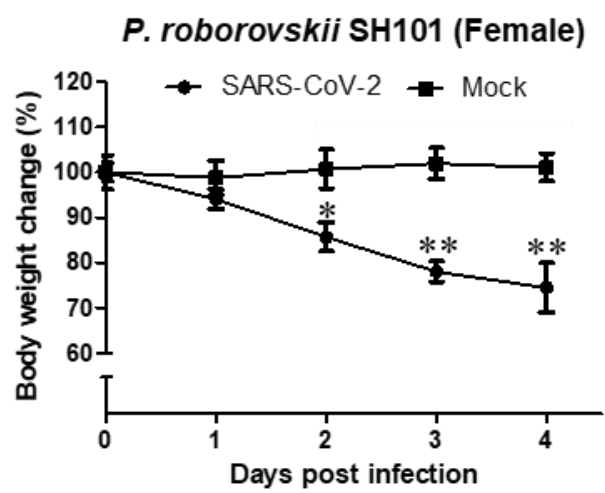

B

P. roborovskii SH101 (Male)

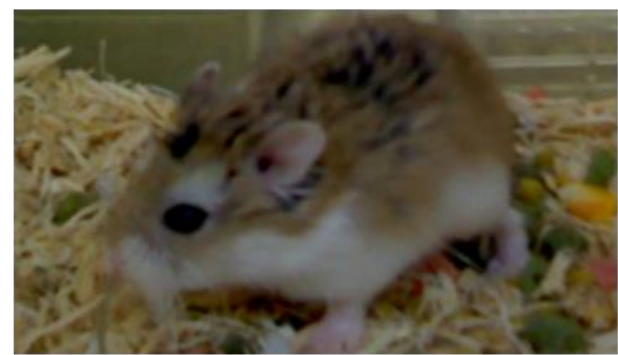

D

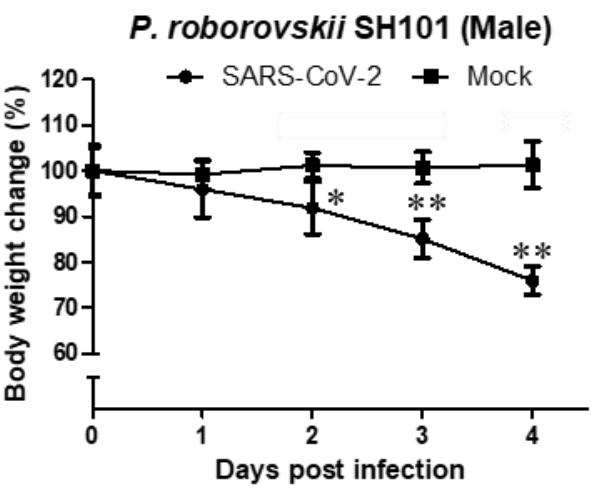

$\mathbf{E}$
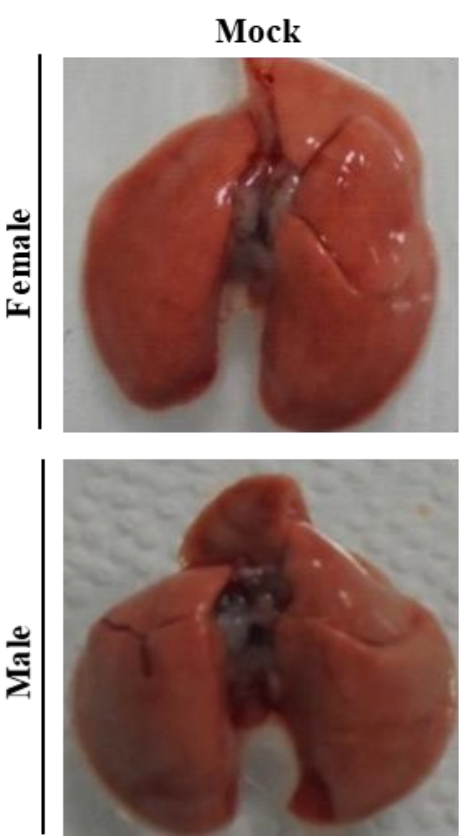

P. roborovskii SH101 + SARS-CoV-2
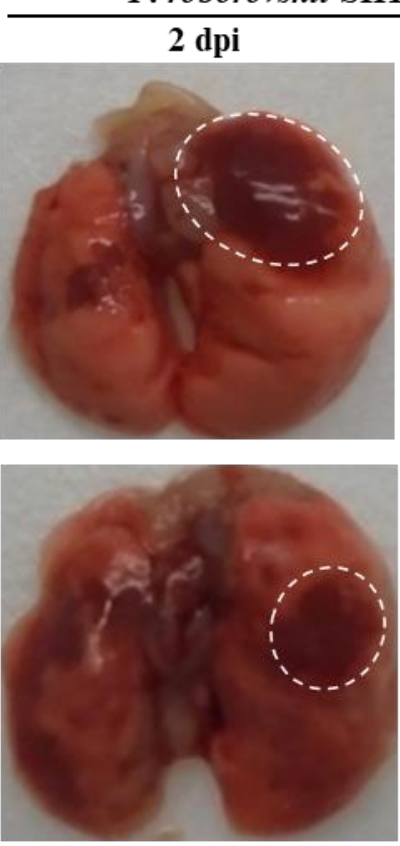

Fig 1
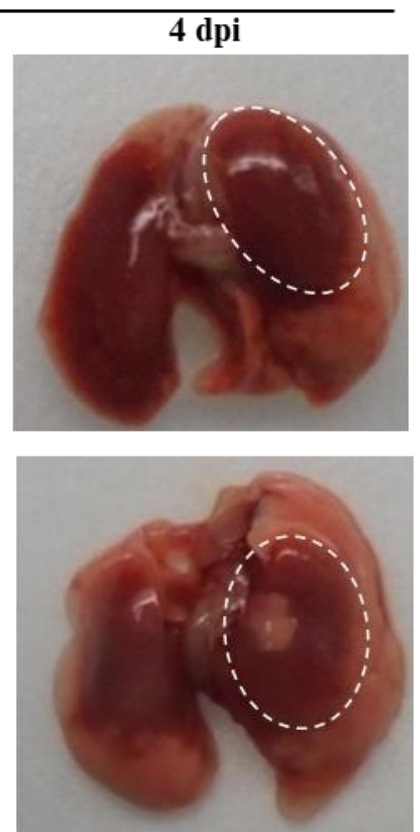
496 Fig 1. Identification of a small animal model for SARS-CoV-2 infection representing most

497 clinical features of COVID-19. (A, B) The photographic image of adult female (A) and male (B)

498 Phodopus roborovskii SH101, a laboratory inbred strain. (C, D) The body weight changes for

499 female (C) and male (D) P. roborovskii SH101 post-infection of SARS-CoV-2. The body weights

500 were measured daily for 5 days (up to 4 dpi) $(n=6)$. Data are presented as mean \pm SD. The

501 statistical significances are marked on the graphs as $* P<0.05$ and $* * P<0.01$. (E) The

502 photographic images of the dissected lungs of the SARS-CoV-2-infected hamster with right-

503 predominant pneumonia indicated as white dotted circles. 
$\mathbf{A}$
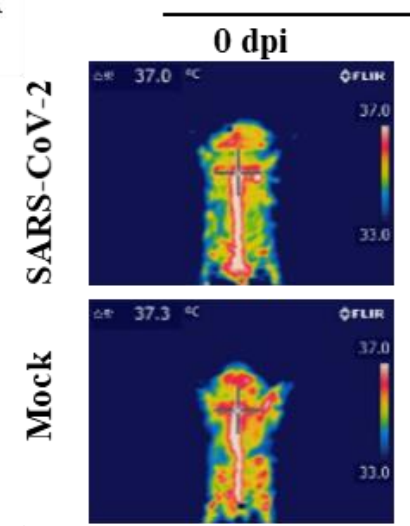

B
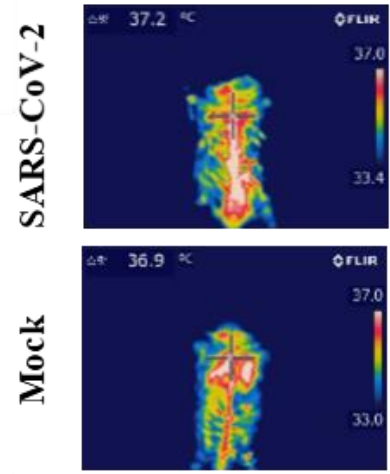

C
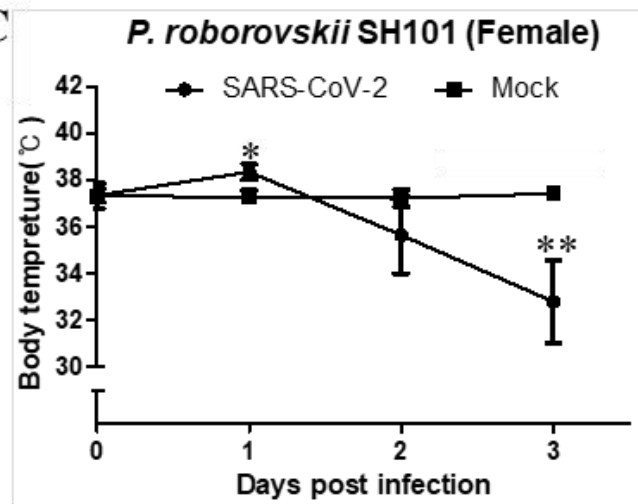

P. roborovskii SH101
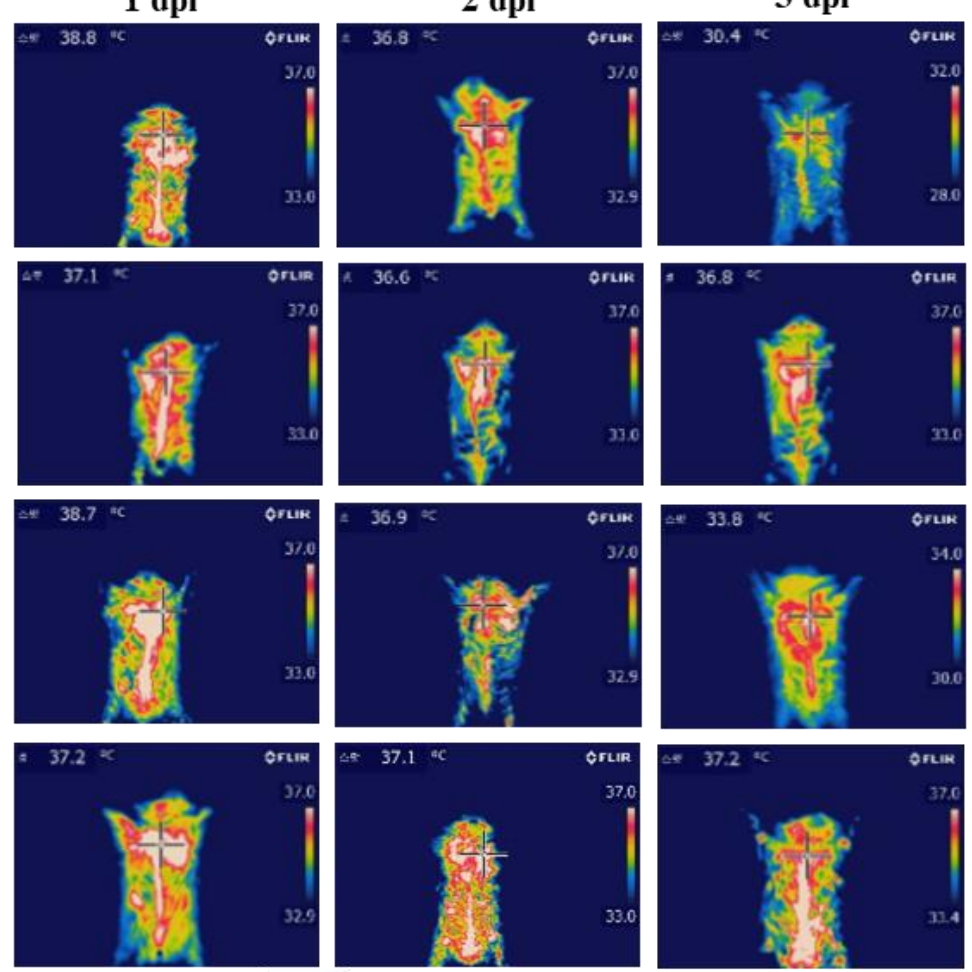

D
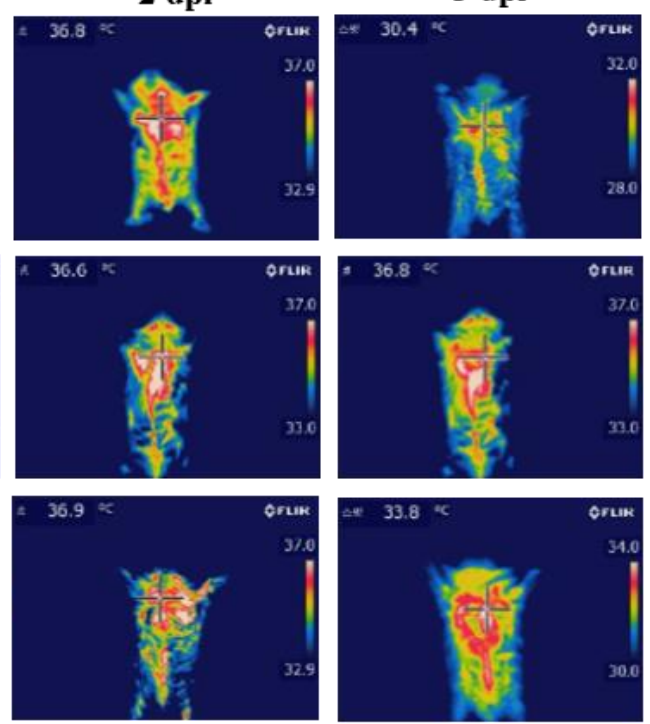

D
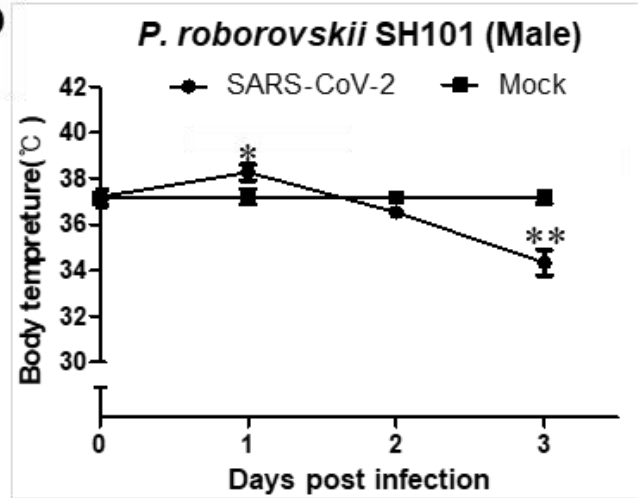
506 Fig 2. The body temperature changes of $P$. roborovskii SH101 infected with SARS-CoV-2. (A,

507 B) The representative infrared thermographic images of the female (A) and male (B) P. roborovskii

508 SH101 for 0, 1, 2, 3 days post-infection of SARS-CoV-2. (C, D) The body surface temperatures

509 on the chest, as close as possible to the lung, of the female (C) and male (D) P. roborovskii SH101

510 hamster infected with SARS-CoV-2 $(n=6)$. The body temperatures were measured by selecting

511 the highest temperature spot on the thermal images and are presented as mean \pm SD. The statistical

512 significances are marked on the graphs as $* P<0.05$ and $* * P<0.01$. 

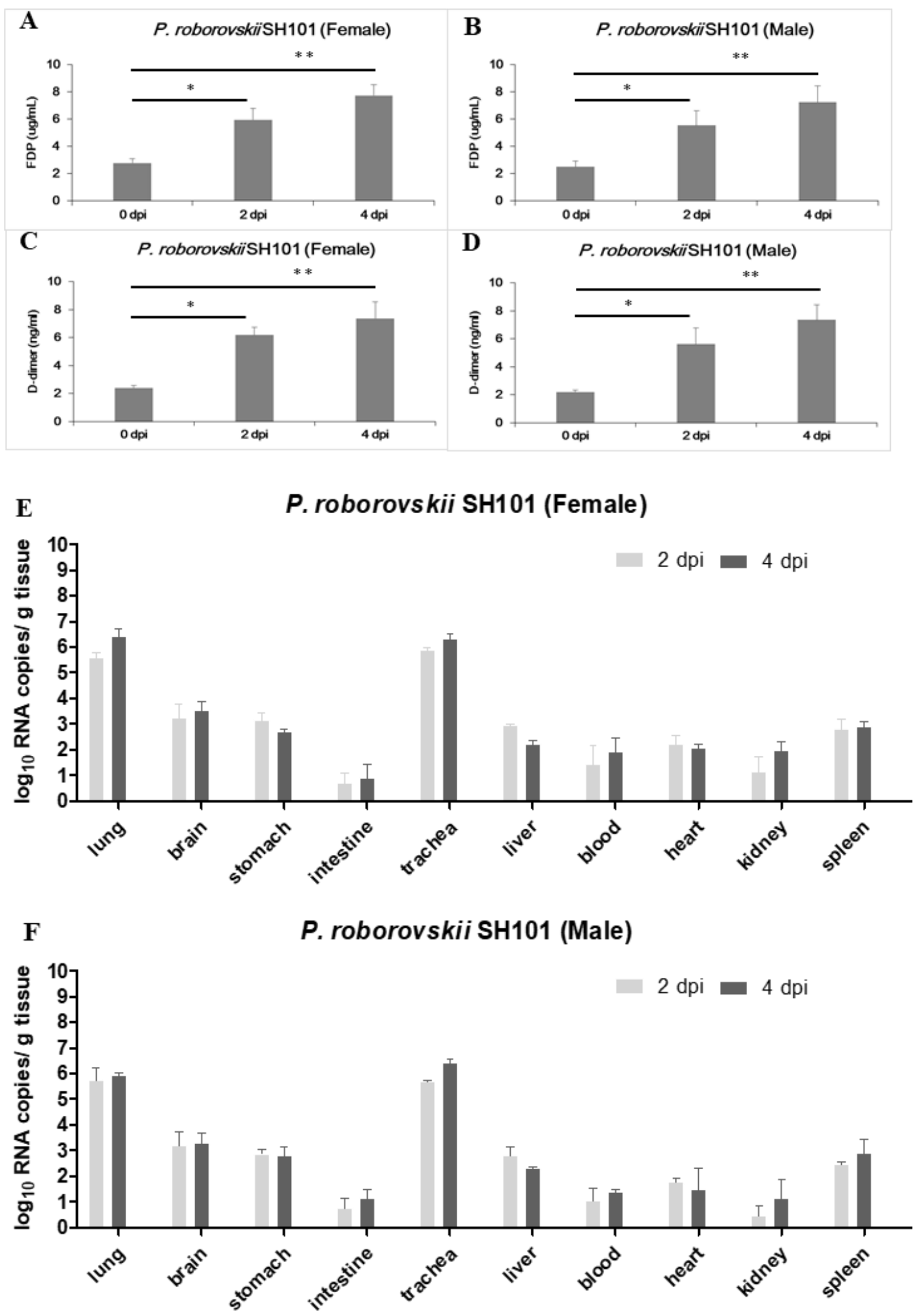
516 Fig 3. Thrombosis and viral replication in $\boldsymbol{P}$. roborovskii SH101 infected with SARS-CoV-2.

517 (A, B) The levels of fibrin degradation products (FDP) in the plasma of the female (A) and male

518 (B) P. roborovskii SH101 hamsters at 2 and 4 dpi of SARS-CoV-2 (n=3). (C, D) The levels of D-

519 dimer in the plasma of the female (C) and male (D) P. roborovskii SH101 hamsters at 2 and 4 dpi

520 of SARS-CoV-2 $(\mathrm{n}=3)$. (E, F) The viral RNA levels in the lung, brain, stomach, intestine, trachea,

521 liver, blood, heart, kidney, and spleen of the female (E) and male (F) P. roborovskii SH101

522 hamsters measured by RT-qPCR at 2 and 4 dpi of SARS-CoV-2. Data are presented as mean \pm SD

$523(\mathrm{n}=3)$. The statistical significances are marked on the graphs as $* P<0.05$ and $* * P<0.01$. 
bioRxiv preprint doi: https://doi.org/10.1101/2021.03.10.434891; this version posted March 11, 2021. The copyright holder for this preprint (which was not certified by peer review) is the author/funder, who has granted bioRxiv a license to display the preprint in perpetuity. It is made available under aCC-BY 4.0 International license.

$\mathbf{A}$
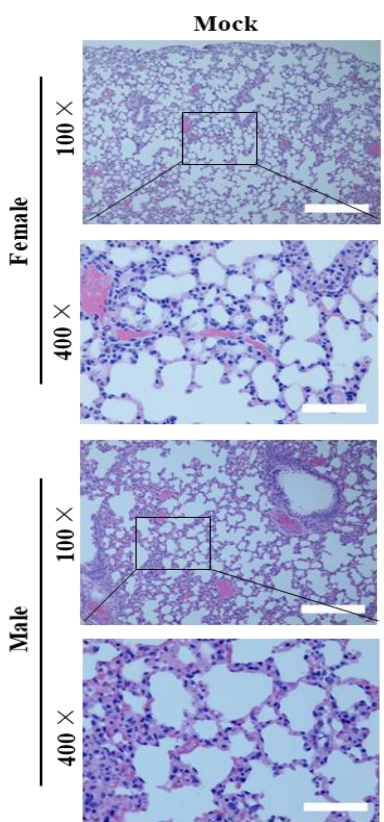

B
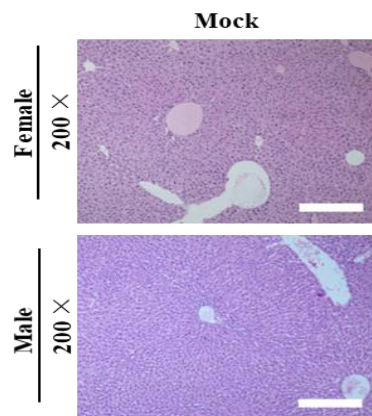

C
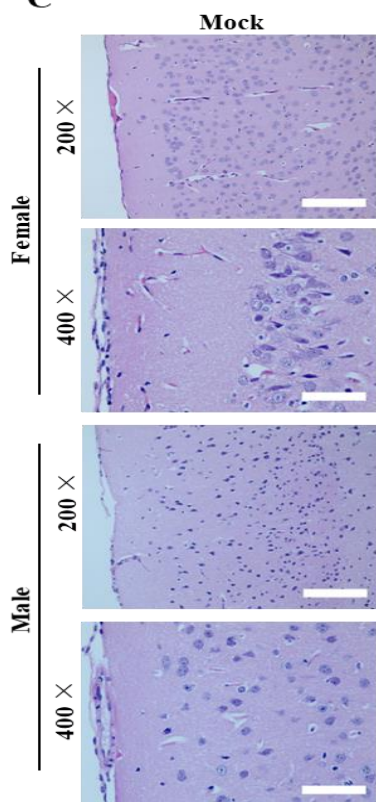

P. roborovskii SH101 + SARS-CoV-2 (Lung)
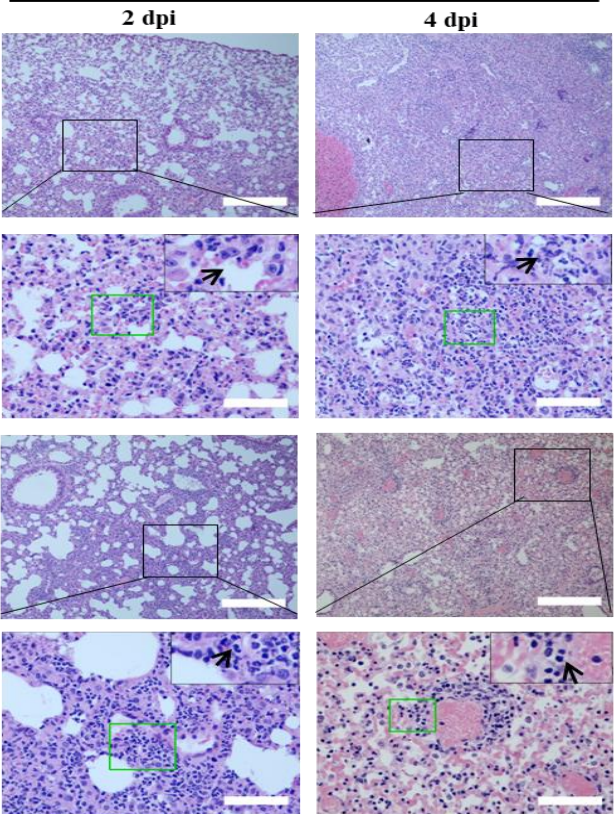

P. roborovskii SH101 + SARS-CoV-2 (Liver)
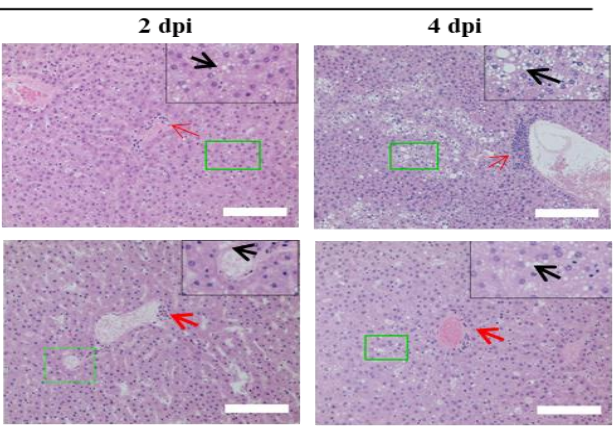

P. roborovskii SH101 + SARS-CoV-2 (Brain)
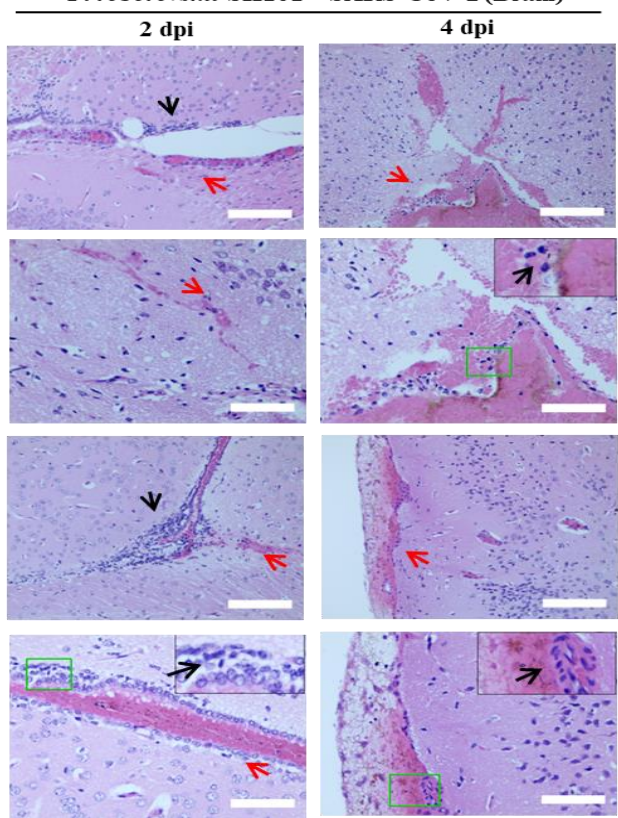
527 Fig 4. Histological examination of $P$. roborovskii SH101 infected with SARS-CoV-2. (A) The

528 representative images of the H\&E-stained histological sections of the lungs of P. roborovskii

529 SH101 at 2 and 4 dpi of SARS-CoV-2. Multifocal interstitial pneumonia with thickened alveolar

530 septa (green frame) and pinkish fibrinous infiltration of inflammatory cells (black arrows) are

531 indicated. (B) The representative images of the H\&E-stained histological sections of the livers of

532 P. roborovskii SH101 at 2 and 4 dpi of SARS-CoV-2 showing pathologies. Focal and intraportal

533 lymphoid cell aggregation and multifocal fatty changes are indicated by red and black arrows,

534 respectively. (C) The representative images of the H\&E-stained histological sections of the brains

535 of $P$. roborovskii SH101 showing pathologies-at 2 and 4 dpi. Subarachnoid hemorrhage and

536 lymphocyte focal infiltration are indicated by red and black arrows, respectively. The scale bars

537 represent $100 \mu \mathrm{m}$ for $100 \times, 50 \mu \mathrm{m}$ for $200 \times$, and $20 \mu \mathrm{m}$ for $400 \times$. 
A hACE-transgenic mice

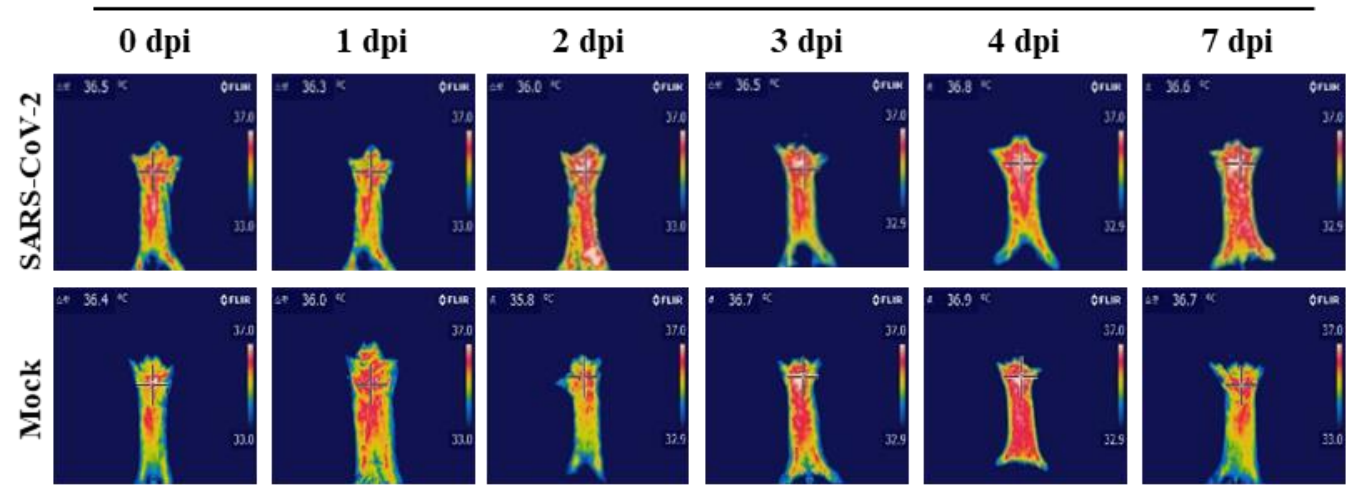

B

Syrian golden hamsters

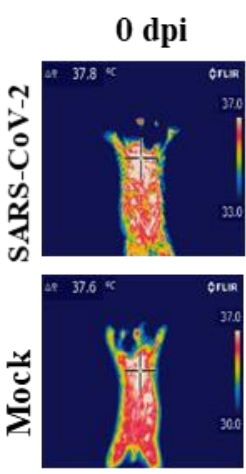

\section{1 dpi}
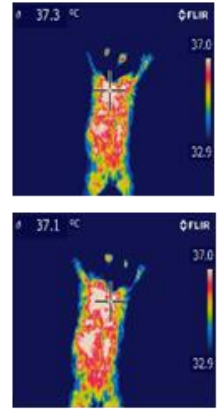

C

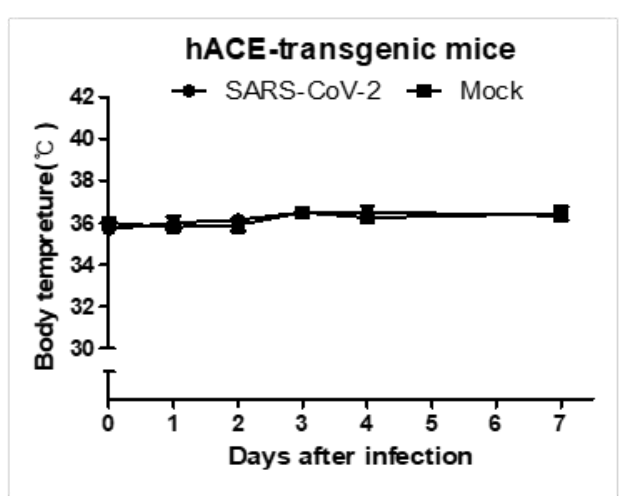

2 dpi
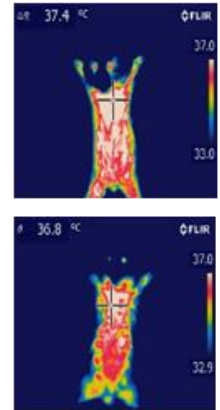

D
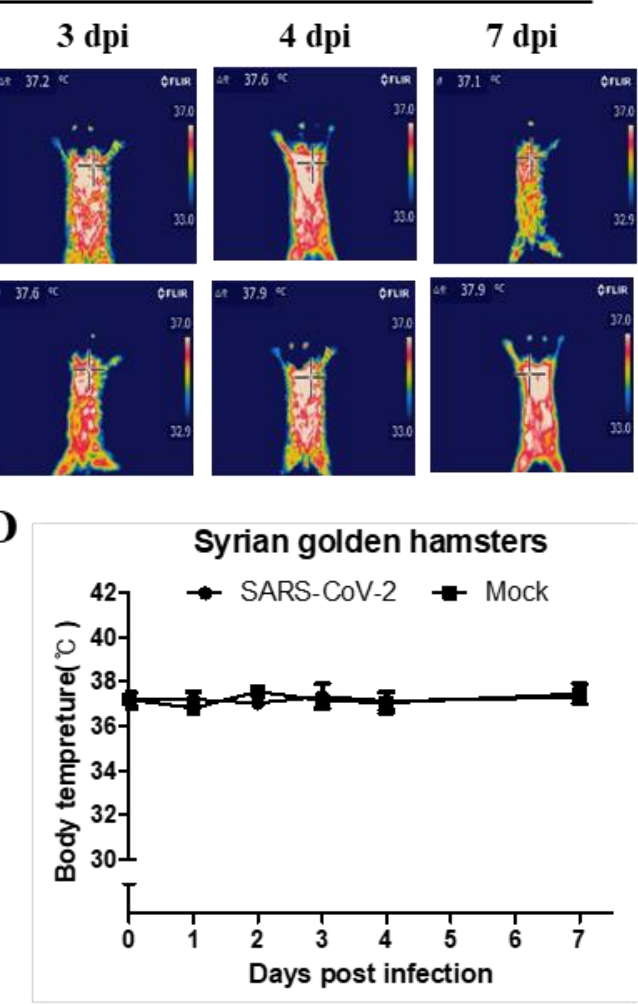

\section{7 dpi}
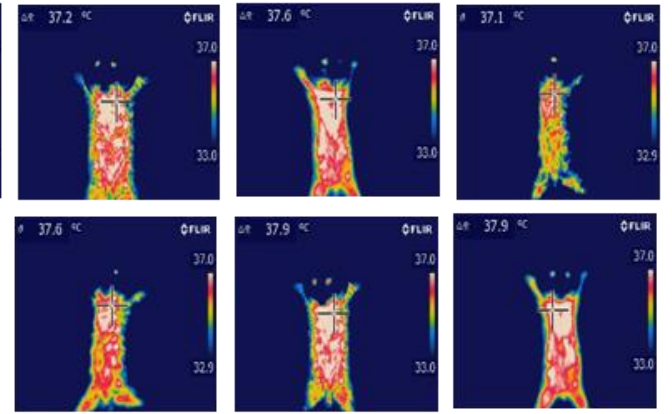

\section{.}


540 Fig 5. The body temperature changes of the hACE-transgenic mice and Syrian golden

541 hamsters after infection of SARS-CoV-2. (A, B) The representative infrared thermographic

542 images of the male hACE-transgenic mice (A) and male Syrian golden hamsters (B) at the

543 indicated dpi of SARS-CoV-2. (C, D) The body temperatures on the chest, as close as possible to

544 the lung, of the male hACE transgenic mice (C) and male Syrian golden hamster (D) at the

545 indicated dpi $(n=6)$. The body temperatures were measured by selecting the highest temperature

546 spot on the thermal images and are presented as mean \pm SD. 


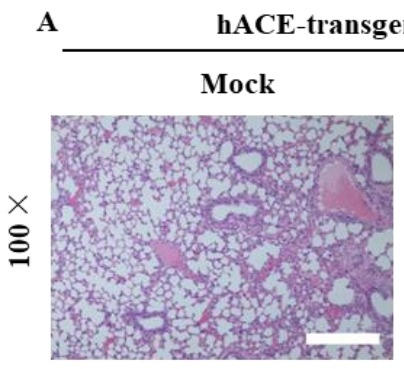

B Syrian golden hamsters (Lung)

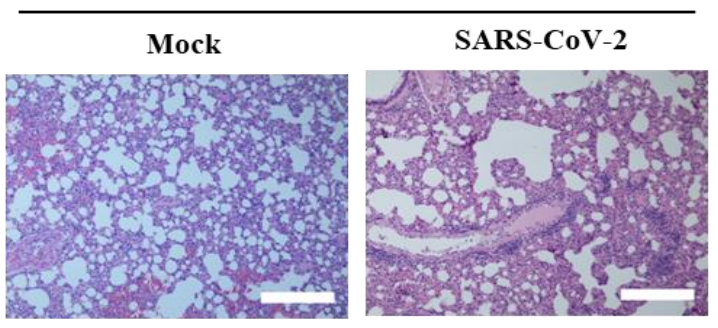

C

hACE-transgenic mice (Liver)

D

Syrian golden hamsters (Liver)

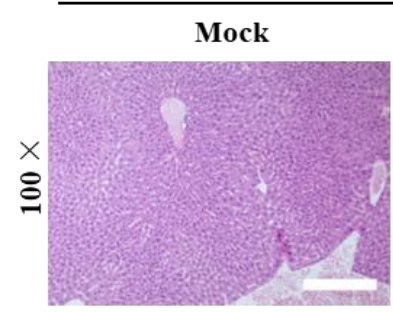

SARS-CoV-2
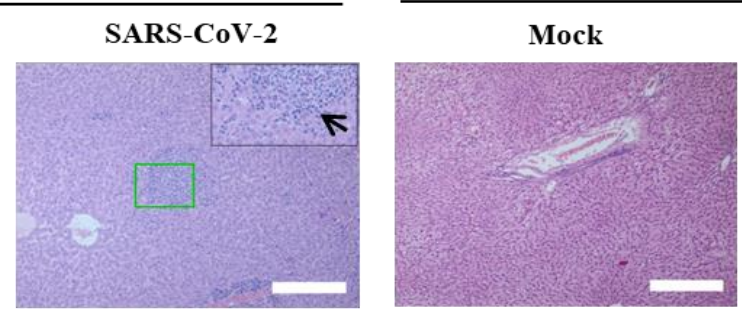

SARS-CoV-2

E

hACE-transgenic mice

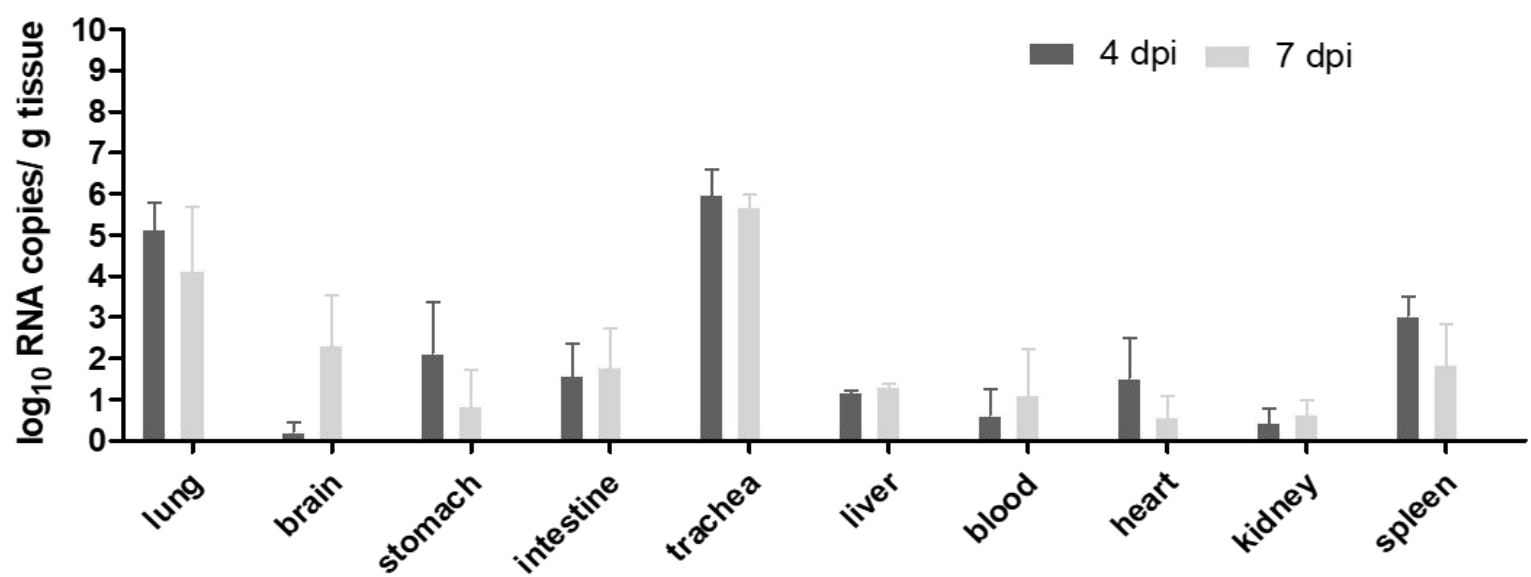

$\mathbf{F}$

Syrian golden hamsters

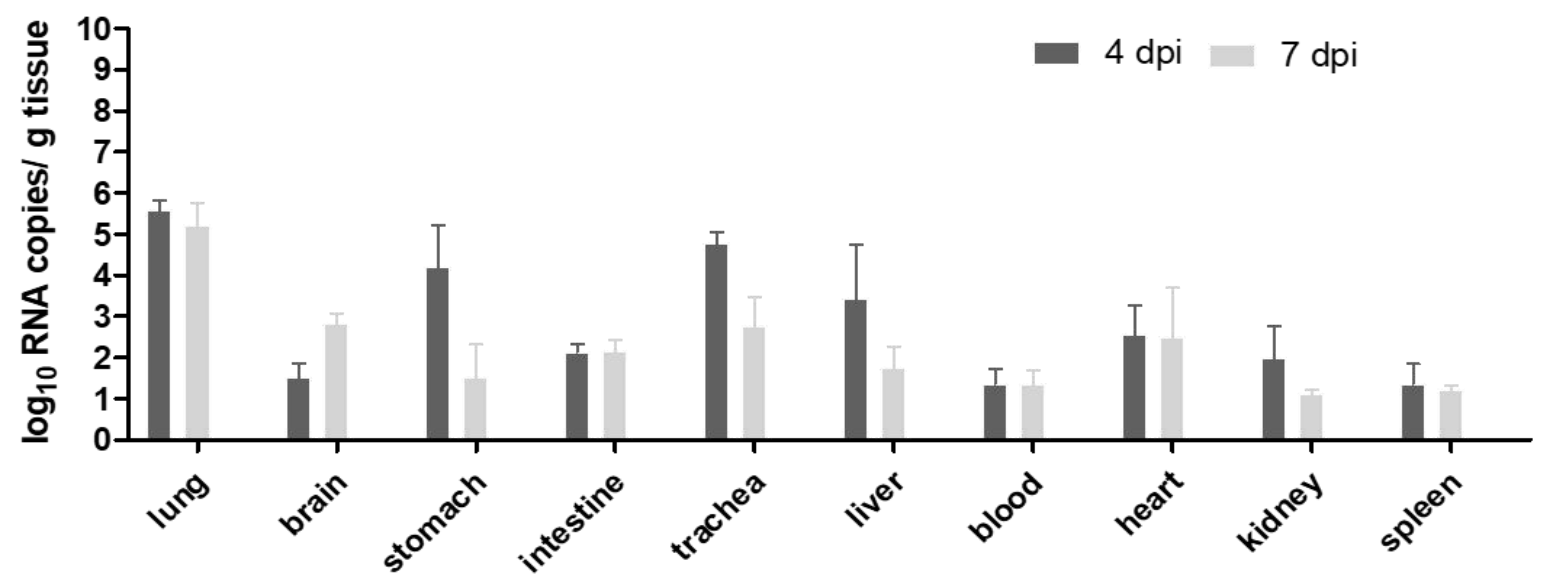


550 Fig 6. Histological examination and SARS-CoV-2 replication in the hACE-transgenic mice

551 and Syrian golden hamsters infected with SARS-CoV-2. (A-D) The representative images of

552 the H\&E-stained histological sections of the lungs $(\mathrm{A}, \mathrm{B})$ and the livers $(\mathrm{C}, \mathrm{D})$ of the hACE-

553 transgenic mice and Syrian golden hamsters at 7 dpi of SARS-CoV-2 showing pathologies.

554 Multifocal interstitial pneumonia with thickened alveolar septa (green frame) and infiltration of

555 inflammatory cells (black arrows) are indicated. (E, F) The viral RNA levels in the primary organs,

556 lung, brain, stomach, intestine, trachea, liver, blood, heart, kidney, and spleen, of the male hACE-

557 transgenic mice (E) and the male Syrian golden hamsters (F) measured by RT-qPCR at 4 and 7

558 dpi of SARS-CoV-2. Data are present as mean \pm SD $(n=3)$. The scale bars represent $100 \mu \mathrm{m}$ for

$559100 \times$ magnifications. 